\title{
Serum MiRNA Biomarkers serve as a Fingerprint for Proliferative Diabetic Retinopathy
}

\author{
Shao Qing ${ }^{\mathrm{a}}$ Songtao Yuan ${ }^{\mathrm{a}}$ Chen Yun ${ }^{\mathrm{b}}$ Hang Hui ${ }^{\mathrm{a}}$ Pingan Mao ${ }^{c}$ Fan Wen ${ }^{\mathrm{a}}$ \\ Yuzhi Ding ${ }^{a}$ Qinghuai Liu ${ }^{\mathrm{a}}$ \\ aDepartment of Ophthalmology, The First Affiliated Hospital of Nanjing Medical University, Nanjing \\ bDepartment of Microbiology and Immunology, Nanjing Medical University, Nanjing, China; \\ 'Department of Ophthalmology, The Second People's Hospital, Changzhou, China
}

\section{Key Words}

PDR • NPDR • Serum miRNA • Dynamic monitoring, signature

\begin{abstract}
Background: Diabetic retinopathy (DR) is a retinopathy resulting from diabetes mellitus (DM) which was classified into non-proliferative DR (NPDR) and proliferative DR (PDR). Without an early screening and effective diagnosis, patients with PDR will develop serious complications. Therefore, we sought to identify special serum microRNAs (miRNAs) that can serve as a novel non-invasive screening signature of PDR and test its specificity and sensitivity in the early diagnosis of PDR. Methods: In total, we obtained serum samples from 90 PDR cases, 90 matched NPDR patients and 20 controls. An initial screening of miRNA expression was performed through TaqMan Low Density Array (TLDA). The candidate miRNAs were validated by individual reverse transcription quantitative real-time PCR (RT-qPCR) arranged in an initial and a twostage validation sets. Moreover, additional double-blind testing was performed in 20 patients clinically suspected of having DR to evaluate the diagnostic value and accuracy of the serum miRNA profiling system in predicting PDR. Results: Three miRNAs were significantly increased in patients with PDR compared with NPDR after the multiple stages. The areas under the receiver operating characteristic $(R O C)$ curves of the validated three-serum miRNAs signature were $0.830,0.803$ and 0.873 in the initial and two validation sets, respectively. Combination of miR-21, miR-181c, and miR-1179 possessed a moderate ability to discrimination between PDR and NPDR with an area under ROC value of 0.89 . The accuracy rate of the three-miRNA profile as PDR signature was $82.6 \%$. Conclusions: These data provide evidence that serum miRNAs have the potential to be sensitive, cost-effective biomarkers for the early detection of PDR. These biomarkers could serve as a dynamic monitoring factor for detecting the progression of PDR from NPDR.
\end{abstract}




\section{Introduction}

Diabetic retinopathy (DR) is resulting from diabetes mellitus (DM), one of the major complications of DM and nearly all people with type 1 and more than half with type 2 diabetes develop retinopathy [1]. It remains the leading cause of legal blindness in the workingage population in Western societies [2] and is a result of multiple pathogenetic processes caused by hyperglycemia and abnormalities of insulin signaling pathways [3], leading to retinal microvascular defects and neuroretinal dysfunction and degeneration [4]. DR is classified into non-proliferative DR (NPDR) and proliferative DR (PDR) [5]. NPDR is characterized by microaneurysms and hemorrhages, vascular changes such as venous beading and loops, intraretinal microvascular abnormalities (IRMA), and cotton-wool spots [6]. PDR is characterized by neovascularization on the disc or elsewhere and can lead to severe visual loss through vitreous hemorrhage, tractional retinal detachment and neovascular glaucoma [7]. Although significant progress has been made, molecular mechanisms underlying the pathogenesis of PDR are still not fully understood; current treatments have resulted in only a partial reduction in this risk. There is still no efficient prevention or treatment.

Although some studies have sought to identify risk factor for the development of PDR in the early stages $[8,9]$, no clinically significant single parameter has been identified. New methods to identify NPDR patients at risk to develop PDR are needed. It is quite essential to explore novel biomarkers with more high sensitivity and specificity for the early detection of PDR for preventing visual loss.

MicroRNAs (miRNAs) are a class of highly conserved 19-25 nucleotide noncoding RNAs that regulate gene expression at the posttranscriptional level $[10,11]$. Recently, miRNAs have been demonstrated to play an important role in diabetes and its complications [12], regulating multiple biological pathways closely related to DR [13]. Although current studies have revealed a link between the expression of miRNAs and the development of DR, these studies mainly focused on miRNAs expressed in tissues of animals model. No studies have specifically examined the role of miRNAs in DR patients. The global miRNA pattern in the sera of DR patients has not been determined. The expression of miRNAs in serum, serum, semen, and other body fluids, particularly serum miRNAs, are abundant, quiet stable [14]. Moreover, serum-based screening is easier, non-invasive and more affordable. Recent studies have demonstrated that miRNAs are closely related to various diseases including Cancers, Male Infertility, Gestational Diabetes Mellitus, Idiopathic Nephrotic Syndrome, etc. Circulating miRNAs can serve as biomarkers for various diseases $[15,16]$. These findings indicate that there may be one or more special serum miRNAs expression pattern in PDR that could serve as a novel non-invasive approach for early detection of PDR.

In order to study the relationship between serum miRNA and the occurrence and development of PDR, we compared the levels of miRNAs in serum of NPDR patients and PDR patients. We analyzed circulating miRNA expression differences of the two groups through RT-PCR-based TaqMan low density Array (TLDA). This undoubtedly laid a solid foundation for further research of warning effect of circulating miRNA for PDR's occurrence and development.

\section{Materials and Methods}

\section{Study design, group subjects}

This study was approved by the Institutional Ethics Committee of The First Affiliated Hospital of Nanjing Medical University, and a written informed consent was also obtained from each participant. All 180 patients underwent ophthalmologic examination including visual acuity, slit lamp examination, fundus contact lens examination and fluorescein angiography. Another 20 volunteers from two independent group without DM or other malignant disease was included as the control group (each 10 samples defined as control1 and control2). A multi-stage, case-control study was designed to identify a serum miRNA profile as a signature for distinguishing PDR from NPDR (Fig. 1). In the screening stage, 5 PDR and 5 matched NPDR serum sam- 
Fig. 1. Systematic overview of the workflow.

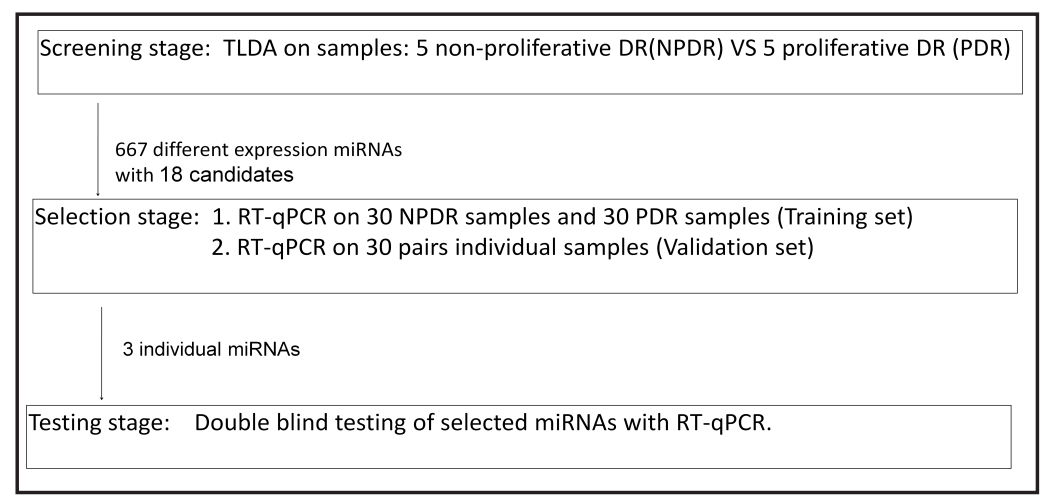

ples were subjected for TLDA to identify the miRNAs that were differentially expressed between PDR and NPDR. Thereafter, we performed individual RT-qPCR in the training phase to further filter signals of the screened miRNAs. Subsequently, we perfected the number of serum miRNAs included as the PDR signature by a two-stage validation, including an internal and an external validation, to confirm the results computed by the front stage. For internal validation, 30 cases and 30 non-PDR controls (Group1) were tested, whereas the external validation phase used serum samples from an additional 30 PDR and 30 NPDR (Group2). We also analyzed another 10 PDR cases and 10 PDR (Group1) in a blinded fashion (the investigators performing the molecular analysis on the blood samples were blinded to the patients' clinical diagnosis) to validation of the diagnostic capability of the candidate miRNAs. The protocols, including the diagnosis procedure and serum collection methods, were identical in the two hospitals.

\section{RNA isolation and RT-qPCR assay}

Up to $3 \mathrm{ml}$ of whole blood from each fasting participant was collected with cases and non-PDR controls prior to any therapeutic procedures, the samples used in our study was obtained from the blood samples collected with non-anticoagulant tube. Each blood sample was immediately centrifuged at 3000g for $10 \mathrm{~min}$ at room temperature and then centrifuged at $10000 \mathrm{~g}$ for $5 \mathrm{~min}$ at $4{ }^{\circ} \mathrm{C}$. MiRNAs were reverse-transcribed with the TaqMan MicroRNA Reverse Transcription Kit (Life Technologies) as described by the manufacturer.

MiRNA-specific TaqMan MicroRNA Assays (Life Technologies) was used for the serum miRNAs. The data obtained were calculated by the $\Delta$ Cq method as described before [17]. The relative expression corresponded to the $2^{\triangle \mathrm{Cq}}$ value. For the analysis of the expression levels of miRNAs, both the internal reference (U6) and external normalization cel-miR-39 was applied for normalization.

TaqMan low density array

MiRNA profiling was performed using TLDA (v2.0) according to the manufacturer's recommended protocol (Life Technologies).

\section{Statistical analysis}

The miRNA data were expressed as the median (interquartile interval), and other variables were expressed as the mean (SD). Chi-square test analysis of variance was used to evaluate statistical differences in demographic and clinical characteristics. The nonparametric Mann-Whitney U-test was used to compare differences in serum miRNA expression which are presented as box plot of the median and range of logtransformed relative expression level. The hierarchical cluster analysis (Average linkage) was applied by using Cluster software and Treeview. Risk score analysis was performed to investigate the effectiveness of the three-serum miRNA signature for PDR predicting. Frequency tables and ROC curves were then used to evaluate the diagnostic effect of the profiling, to find the appropriate cutoff point, and to validate the procedure and cutoffs in the next validation sample set.ROC curves were then used to evaluate the diagnostic effects of the profiling and to find the appropriate cutoff point, and to validate the procedure and cutoffs in the next validation sample set. First, the risk score of each serum sample in the training set was calculated, as the basis of their risk scores and a set cut off, serum samples were then divided into a high-risk group, representing the possible PDR, and a low-risk group, representing the predicted NPDR. At the optimal cutoff 


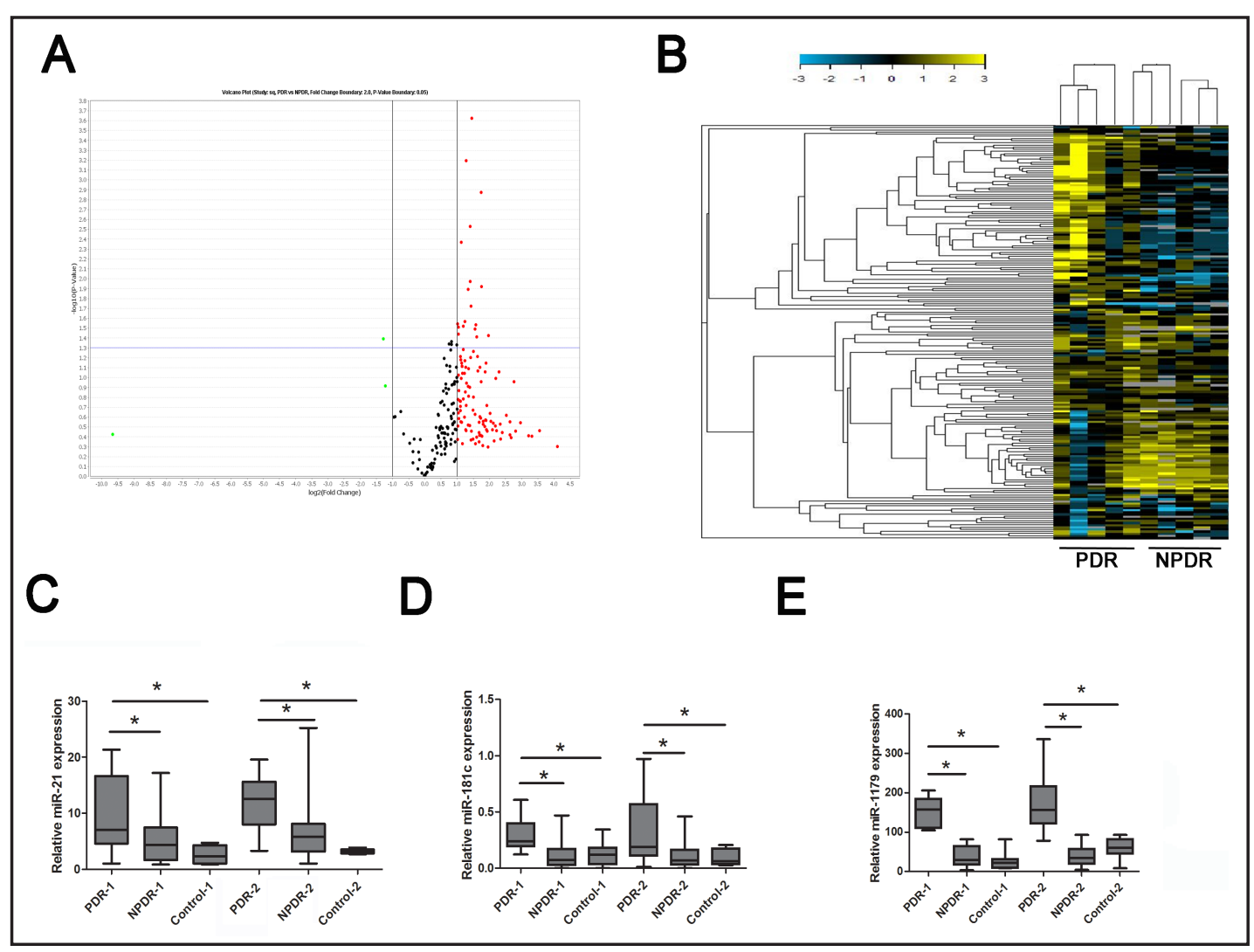

Fig. 2. MiR-21, 181c and 1179 was confirmed as the candidate miRNAs according to the RT-PCR-based TLDA. A: The volcano plot of the aberrant expressed miRNAs detected in the RT-PCR-based TLDA. The red plot indicated the up-regulation miRNAs while the green indicated the down-regulated miRNAs. B: Cluster analysis of the different expression of the miRNAs. C-E: Increased level of miR-21, 181c and 1179 was confirmed by RT-PCR in groups. Data were presented as box plot of the median and range of log-transformed relative expression level. The top and bottom of the box represent the seventy-fifth and twenty-fifth percentile. * indicated $\mathrm{P}<0.05$.

value with the value of sensitivity + specificity considered to be maximal. Statistical analysis was performed using STATA 9.2, and presented with GraphPad Prism 5.0 software. Results were considered statistically significant at $P<0.05$.

\section{Results}

Selection and validation phase

In order to gain an expression profile of serum miRNAs that is specific for PDR, the TLDA was used to identify the differentially expressed miRNAs in 5 PDR cases and 5 NPDR in the initial screening phase (Fig. 2A, B). Among 667 miRNAs analysed, 18 up-regulated miRNAs were identified which satisfy the threshold that at most 30 of CT value and at least 8 average fold change by TLDA in both two group for further individual validation (Table 1). The detailed data of the TLDA has been uploaded in the ArrayExpress database (www.ebi. ac.uk/arrayexpress) under accession number E-MTAB-2559.

We further examined the18 candidate miRNAs by RT-qPCR in a training sample set (30 patients with PDR and 30 patients with NPDR). We have detected the all the 18 up-regulation miRNAs and the only down-regulation miRNA in the following stages, and found that the down-regulated miRNA indicated no difference between the PDR and NPDR. As the difference in miR-21, miR-181c and miR-1179 expression level between PDR cases and NPDR 
Table1. Different expression of the miRNAs detected according to the miRNA expression microarray

\begin{tabular}{cllcc}
\hline & Assay & Type & PDR (RQ) & PDR (P-Value) \\
\hline Up-regulated & hsa-miR-1179-002776 & Target & 3.0509 & 0.0386 \\
Up-regulated & hsa-miR-1255B-002801 & Target & 2.2841 & 0.0302 \\
Up-regulated & hsa-miR-148a-4373130 & Target & 2.0583 & 0.0364 \\
Up-regulated & hsa-miR-181c-4373115 & Target & 2.7274 & $2.00 \mathrm{E}-04$ \\
Up-regulated & hsa-miR-21-4373090 & Target & 2.3618 & 0.027 \\
Up-regulated & hsa-miR-30a-5p-000417 & Target & 2.0209 & 0.0286 \\
Up-regulated & hsa-miR-363-4378090 & Target & 2.6437 & 0.003 \\
Up-regulated & hsa-miR-374a\#-002125 & Target & 2.0603 & 0.031 \\
Up-regulated & hsa-miR-423-5p-4395451 & Target & 2.6968 & 0.0189 \\
Up-regulated & hsa-miR-494-4395476 & Target & 2.1839 & 0.0043 \\
Up-regulated & hsa-miR-505\#-002087 & Target & 3.3628 & 0.012 \\
Up-regulated & hsa-miR-520D-3P-002743 & Target & 2.9813 & 0.0292 \\
Up-regulated & hsa-miR-548d-3p-4381008 & Target & 3.9242 & 0.0376 \\
Up-regulated & hsa-miR-576-3p-4395462 & Target & 2.9254 & 0.0323 \\
Up-regulated & hsa-miR-601-001558 & Target & 2.6526 & 0.0107 \\
Up-regulated & hsa-miR-606-001569 & Target & 2.5293 & 0.0128 \\
Up-regulated & hsa-miR-627-4380967 & Target & 3.3543 & 0.0013 \\
Up-regulated & hsa-miR-886-5p-4395304 & Target & 2.4325 & $6.00 \mathrm{E}-04$ \\
Down-regulated & hsa-miR-409-3p-002332 & Target & 0.4103 & 0.0407 \\
\hline & & & &
\end{tabular}

Table 2. Correlation between patients of PDR and NPDR in clinicopathological characteristics $(n=90)$

\begin{tabular}{lcccc}
\hline Characteristics & PDR & NPDR & Control & $\begin{array}{c}\mathrm{p} \\
\text { Chi-squared test p-value }\end{array}$ \\
\hline No. & 90 & 90 & 20 & \\
Age(years) & & & & \\
$<60$ & 55 & 46 & 10 & 0.176 \\
$\geq 60$ & 35 & 44 & 10 & \\
Gender & & & & \\
Male & 48 & 45 & 10 & \\
Female & 42 & 45 & 10 & \\
Time of DM(years) & & & & 0.655 \\
$<5$ & 16 & 17 & - & \\
$\geq 5$ & 74 & 73 & - & \\
\hline
\end{tabular}

was statistically significance which was consistent with the results of RT-PCR-based TLDA (Fig. 2C-D).

To validate the accuracy and specificity of these miRNAs as a PDR potential signature, we also examined their expression levels though an internal and external individual samples (Group1, 30 PDR vs 30 NPDR, Group2, 30 PDR vs 30 NPDR). As shown in Fig. 2C-D, the expression of three miRNAs in serum of PDR were all significantly higher than those in NPDR in the two groups, which agreed with the training set results. As a result of this multiphase testing and analysis, a profile of 3 miRNAs were considered to be the potential signature for PDR.

\section{Risk score and ROC curve analysis}

To assess the diagnostic value of the three-serum miRNA profiling system, we used a risk score formula to calculate the risk score function for cases and non-PDR control samples. The diagnostic sensitivity and specificity of the miRNAs signature for PDR detection were $82 \%$ and $95 \%$, and the positive predictive value and negative predictive value was $93 \%$ and $85 \%$ in the training set, respectively. Similarly, when the same cutoff was used to calculate the risk score of samples from group1 and group2 validation sets, the diagnostic sensitivity and specificity were $82 \%, 84 \%$ (group 1 ) and $86 \%, 89 \%$ (group2), respectively.

ROC curves analyses were conducted to assess the diagnostic sensitivity and specificity of the three miRNAs signature for PDR by using risk score functions (RSFs). The areas under the curve (AUC) were 0.83 (95\% confidence interval (CI), 0.79-1.00), 0.80 (95\% CI, 0.81- 
Fig. 3. ROC analysis of the three potential biomarker for PDR.
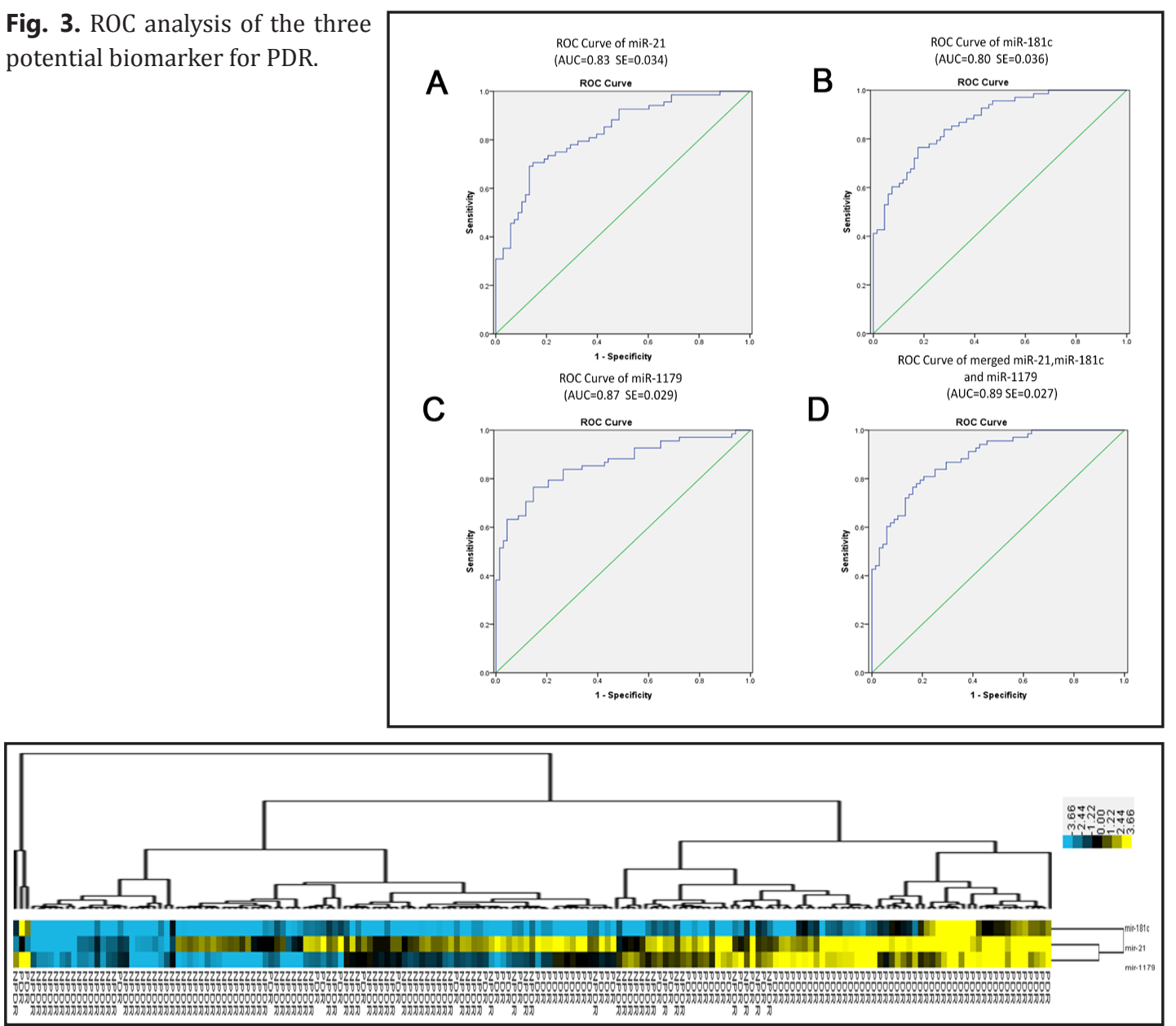

Fig. 4. The unsupervised cluster for analyzing the differential expression of miRNAs between the PDR and NPDR. The results of hierarchical cluster analysis showed a clear separation of the PDR patients from the NPDR patients on the basis of the three- serum miRNA signature.

0.98), 0.87 (95\% CI, 0.86-0.99) and 0.89 (95\% CI, 0.86-0.96) for the serum samples in validation sets (Fig. 3). The results indicated that the three- serum miRNA signature can serve as a novel non-invasive approach for the early screening of PDR from NPDR.

\section{Unsupervised cluster analysis}

The unsupervised cluster method was also performed to analyze the differential expression of miRNAs between the PDR and NPDR. The results of hierarchical cluster analysis showed a clear separation of the PDR patients from the NPDR patients on the basis of the three-serum miRNA signature. As shown in Fig. 4, in the total samples, only 15 out of 90 NPDR cases samples and 10 out of 90 PDR samples were incorrectly classified.

\section{Double-blind test}

Another 20 serum samples (10 PDR and 10 NPDR) were tested in a double-blind fashion to validate the predictive ability of the three miRNA-based signatures for PDR diagnosis. We used the same risk score formula to analyze the expression of the 3 miRNAs in those serum samples and classifying them into a high-risk group and a low-risk group. On the basis of the pathologic diagnosis, the accuracy rate of the three-miRNA profile as PDR signature was $82.6 \%$ 


\section{Discussion}

This study demonstrates a new biomarker for detecting PDR in DR patients for the first time. We have validated the three miRNAs including miR-21, miR-181c and miR-1179 in predicting PDR out of NPDR which is very important for detection the progression of patients with NPDR developing to PDR.

DR is a leading cause of blindness in the world. which is the most common chronic microvascular complication associated with DM [18]. Abnormal retinal blood vessel growth and angiogenesis are the major causes of diabetes-related loss of vision. Vascular endothelial growth factor (VEGF) and pigment epitheliumderived factor (PEDF) are important factors for angiogenesis condition [19]. DR is a highly specific vascular complication and a sightthreatening problem related to diabetes. PDR is characterized by gradually progressive alterations in the retinal microvasculature, leading to retinal hypo perfusion, increased vascular permeability, and pathologically intraocular proliferation of retinal vessels [20]. Thus, a novel factor to monitor the progression of the proliferation of vascular endothelial is very important for both the prevention and the updating of the therapy.

It has been reported that the serum miRNA expression profiles for various diseases may serve as fingerprints for disease detection [21]. A serum miRNA-based signature would make it possible to comprehensively analyze PDR without or less used of other invasive procedures. This is the first investigation that demonstrates the serum miRNA profile could be used as potential biomarker of PDR. We identified three PDR-associated miRNAs, including miR-21, miR-181c and miR-1179. Our results indicate that PDR is associated with a combination of multiple serum miRNAs which is a more comprehensive indicator for the disease detection. In order to investigate the potential mechanism of miRNAs in the pathogenesis of PDR, we searched the target genes associated with miR-21, miR-181c and miR-1179. We found that miR-21 was reported inducing tumor angiogenesis through targeting PTEN, leading to activate AKT and ERK1/2 signaling pathways, and thereby enhancing HIF-1 $\alpha$ and VEGF expression [22], furthermore, miR-21 protected endothelial cell against high glucoseinduced endothelial cytotoxicity [23]. These indicated that miR-21 was highly associated with the angiogenesis with the microenvironment of high glucose. Besides, miR-181c has been confirmed with high level in vein endothelial cells treated in a diabetic-like environment indicating that miR-181c might be associated with vascular proliferation in high glucose [24].

From a clinical point of view, the miRNA biomarker selected in our study for the monitoring of PDR might be useful for indicating the clinical typing of DR which is a great help to decide the way of the treatment. Furthermore, for as a single diagnostic method, the biomarker in our study presented a high sensitivity and specificity, nevertheless, given that the express fluctuations of serum miRNA, analysis with a panel of multiple diagnosis methods might be a solution to the problem of PDR detection.

In conclusion, we have identified a unique miRNA biomarker for early screening of PDR, which may serve as a novel non-invasive approach for diagnosis and dynamic monitoring of PDR.

\section{Acknowledgments}

This study was supported by Natural Science Foundation of China [NSFC 81070743 and [NSFC 81272322] and [NSFC 30901750]. This research project was also supported by National Basic Research Program of China (973 Program, No.2012CB066300 and No.2011CB510200, General Project of the National Natural Science Fund (No. 81170855 and No.81070743, and a Project Funded by the Priority Academic Program Development of Jiangsu Higher Education Institutions (PAPD, JX10231801); Jiangsu Provincial Special Program of Medical Science (BL2014089). The funders had no role in study design, data collection and analysis, decision to publish, or preparation of the manuscript. 


\section{Cellular Physiology and Biochemistry}

Qing et al.: Serum miRNA Biomarker for PDR

\section{Disclosure Statement}

There are no conflicts of interest.

\section{References}

$>1$ Fong DS, Aiello L, Gardner TW, King GL, Blankenship G, Cavallerano JD, Ferris FL 3rd, Klein R: Diabetic retinopathy. Diabetes Care 2003;26:226-229.

-2 Chang YC, Wu WC: Dyslipidemia and diabetic retinopathy. Rev Diabet Stud 2013;10:121-132.

3 Brownlee M: The pathobiology of diabetic complications: A unifying mechanism. Diabetes 2005;54:16151625.

-4 Reiter CE, Gardner TW: Functions of insulin and insulin receptor signaling in retina: Possible implications for diabetic retinopathy. Prog Retin Eye Res 2003;22:545-562.

5 Curtis TM, Gardiner TA, Stitt AW: Microvascular lesions of diabetic retinopathy: Clues towards understanding pathogenesis? Eye (Lond) 2009;23:1496-1508.

6 Antonetti DA, Barber AJ, Bronson SK, Freeman WM, Gardner TW, Jefferson LS, Kester M, Kimball SR, Krady JK, LaNoue KF, Norbury CC, Quinn PG, Sandirasegarane L, Simpson IA: Diabetic retinopathy: Seeing beyond glucose-induced microvascular disease. Diabetes 2006;55:2401-2411. Cheung N, Mitchell P, Wong TY: Diabetic retinopathy. Lancet 2010;376:124-136.

8 Shahin M, El-Diasty AM, Mabed M: Anticardiolipin antibodies in proliferative diabetic retinopathy: An additional risk factor. Saudi J Ophthalmol 2009;23:165-169.

-9 Beranek M, Kankova K, Benes P, Izakovicova-Holla L, Znojil V, Hajek D, Vlkova E, Vacha J: Polymorphism r25p in the gene encoding transforming growth factor-beta (tgf-beta1) is a newly identified risk factor for proliferative diabetic retinopathy. Am J Med Genet 2002;109:278-283.

10 Bartel DP: Micrornas: Genomics, biogenesis, mechanism, and function. Cell 2004;116:281-297.

11 Plasterk RH: Micro rnas in animal development. Cell 2006;124:877-881.

12 Pandey AK, Agarwal P, Kaur K, Datta M: Micrornas in diabetes: Tiny players in big disease. Cell Physiol Biochem 2009;23:221-232.

$\checkmark 13$ Suarez Y, Sessa WC: Micrornas as novel regulators of angiogenesis. Circ Res 2009;104:442-454.

$>14$ Tang W, Li H, Tang J, Wu W, Qin J, Lei H, Cai P, Huo W, Li B, Rehan V, Xu X, Geng Q Zhang H, Xia Y: Specific serum microrna profile in the molecular diagnosis of hirschsprung's disease. J Cell Mol Med 2014

15 Kroh EM, Parkin RK, Mitchell PS, Tewari M: Analysis of circulating microrna biomarkers in plasma and serum using quantitative reverse transcription-pcr (qrt-pcr). Methods 2010;50:298-301.

-16 Liu R, Chen X, Du Y, Yao W, Shen L, Wang C, Hu Z, Zhuang R, Ning G, Zhang C, Yuan Y, Li Z, Zen K, Ba Y, Zhang CY: Serum microrna expression profile as a biomarker in the diagnosis and prognosis of pancreatic cancer. Clin Chem 2012;58:610-618.

17 Wang C, Yang C, Chen X, Yao B, Zhu C, Li L, Wang J, Li X, Shao Y, Liu Y, Ji J, Zhang J, Zen K, Zhang CY, Zhang C: Altered profile of seminal plasma micrornas in the molecular diagnosis of male infertility. Clin Chem 2011;57:1722-1731.

-18 Loukovaara S, Koivunen P, Ingles M, Escobar J, Vento M, Andersson S: Elevated protein carbonyl and hif1alpha levels in eyes with proliferative diabetic retinopathy. Acta Ophthalmol 201 4;92:323-327.

19 Liu J, Xia X, Xiong S, Le Y, Xu H: Intravitreous high expression level of netrin-1 in patients with proliferative diabetic retinopathy. Eye Sci 2011;26:85-90, 120.

20 Gilbert RE: The endothelium in diabetic nephropathy. Curr Atheroscler Rep 2014;16:410.

-21 Cortez MA, Bueso-Ramos C, Ferdin J, Lopez-Berestein G, Sood AK, Calin GA: Micrornas in body fluids--the mix of hormones and biomarkers. Nat Rev Clin Oncol 2011;8:467-477.

22 Liu LZ, Li C, Chen Q, Jing Y, Carpenter R, Jiang Y, Kung HF, Lai L, Jiang BH: Mir-21 induced angiogenesis through akt and erk activation and hif-1alpha expression. PLoS One 2011;6:e19139.

23 Zeng J, Xiong Y, Li G, Liu M, He T, Tang Y, Chen Y, Cai L, Jiang R, Tao J: Mir-21 is overexpressed in response to high glucose and protects endothelial cells from apoptosis. Exp Clin Endocrinol Diabetes 2013;121:425430 .

-24 Zitman-Gal T, Green J, Pasmanik-Chor M, Golan E, Bernheim J, Benchetrit S: Vitamin d manipulates mir181c, mir-20b and mir-15a in human umbilical vein endothelial cells exposed to a diabetic-like environment. Cardiovasc Diabetol 2014;13:8. 